\title{
Molecular cloning of clathrin assembly protein gene (rCALM) and its differential expression to AP180 in rat brain
}

\author{
Hyung-Lae Kim ${ }^{1,3}$ and Sunhee Cho Lee ${ }^{2}$ \\ ${ }^{1}$ Department of Biochemistry and ${ }^{2}$ Department of Physiology, \\ College of Medicine, Ewha Womans University, \\ Seoul 158-056, Korea \\ ${ }^{3}$ Corresponding author: Tel, 82-2-650-5727; Fax, 82-2-650-5791, \\ E-mail, hyung@mm.ewha.ac.kr
}

Accepted 10 November 1999

Abbreviations: CCV, clathrin-coated vesicle; CALM, clathrin assembly protein lymphoid myeloid; PLD, phospholipase D; RT-PCR, reverse transcriptase polymerase chain reaction; $\mathrm{AP}$, adaptor protein

\begin{abstract}
Binding of clathrin assembly protein to clathrin triskelia induces their assembly into clathrin-coated vesicle (CCV) in neurons. The clathrin assembly protein gene (rCALM) was cloned from rat brain cDNA library. rCALM deduced 69 kD molecule has overall $73 \%$ amino acid homology compared with that of AP180 protein. The N-terminal domain, where amino acid sequences are very similar with AP180, harbours binding sites for clathrin and inositides, as well as possible phosphorylation sites, but the proline rich C-terminal domain is different from that of AP180. The mRNA expression of rCALM and AP180 by in situ hybridization histochemistry revealed that the rCALM mRNA was more intensely expressed than that of AP180, and the distribution patterns were different from each other. These results suggest that the rCALM mediates the assembly of clathrin in neural and supporting cells of brain, and regulates the clathrin coated-vesicle formation through phosphorylation and inositide metabolism.
\end{abstract}

Keywords: clathrin-coated vesicle, AP180, CALM, gene cloning, expression

\section{Introduction}

Clathrin-coated vesicles (CCV) are involved in pathways of receptor-mediated intracellular transport and transfer of proteins from trans-Golgi network to pre-lysosomal compartment and recycling of synaptic vesicles (Pearse and Robins, 1990; Keen, 1990). The CCV coat is formed by polymerization of triskelion-shaped clathrin molecules into lattice of clathrin cage, and is catalyzed by the assembly proteins. The major coat protein is a clathrin, which consists of triskelion having three identical $190 \mathrm{kD}$ heavy chains and three 23-27 kD light chains. Coated vesicle also contains one or more of the assembly proteins (Robinson, 1994). The assembly proteins are adaptors, and are believed to link receptors to the clathrin network. The assembly proteins all participate in promoting an assembly of clathrin triskelia into artificial clathrin cage that resemble coated vesicle. To date, six different adaptor proteins (AP) are known: AP-1, AP-2, AP-3, AP4, AP180, CALM, and auxillin. The AP-1, AP-2, AP-3, and AP-4 are tetramer, whereas AP180, CALM, and auxillin are monomer.

AP-1 and AP-2 adaptors mediate clathrin assembly at the trans-Golgi network and plasma membrane, respectively, and play a role in selecting cargo protein in CCVs (Marsh and McMahon, 1999). AP-3 plays a role in trafficking from trans-Golgi network to the lysosome (Simpson et al., 1997). The function of AP-4 associated with transGolgi network was not characterized precisely (Dell'Angelica et al., 1999). AP180 is well studied because it is monomeric protein and neuron specific (Kondury and Roland, 1988; Zhou et al., 1992). AP180 was first discovered as a specific coat component of clathrincoated vesicles from neural tissue and as a phosphoprotein (Stephan et al., 1990). The native protein was shown to associate with clathrin triskelia on equimolar basis and induces clathrin assembly into a homogenous population of $60-70$ $\mathrm{nm}$ coats (Zhou et al., 1993). AP180 is phosphorylated at serine residues (Morris et al., 1990). The disassembly of clathrin from the vesicle coat was promoted by the phosphorylation of AP's (Wilde and Brodsky, 1996). Two known primary functional properties of the AP180 are clathrin assembling and high affinity for specific inositol polyphosphates (Morris et al., 1993; Ye et al., 1995; Norris et al., 1995). The CALM gene was cloned from human leukemia cell line, and was shown to be homologous to the monomeric clathrin adaptor of AP180. But the molecular property of the gene was not characterized. To understand the regulation of CCV formation at a molecular level, I have cloned and characterized the clathrin assembly protein gene from rat brain. In this study, the primary structure of CALM gene (rCALM) from the rat brain cDNA libraries and the differential expression between two monomeric adaptor proteins, AP180 and rCALM, in adult rat brain are presented. 


\section{Materials and Methods}

\section{Materials}

Rat brain cDNA library was purchased from Stratagene. T7 sequencing kit was from US Biochemicals. Exo III deletion kit and Wizard miniprep kit were obtained from Promega. Restriction endonucleases were from Boerhinger Manheim. Nitrocellulose transfer membranes (BA85, 0.45 $\mu \mathrm{m})$ were from Schleicher and Schuell. Other chemicals were the highest purity available.

\section{Cloning of rCALM gene from rat CDNA library}

The rCALM gene was isolated from rat CDNA library using oligonucleotides, 5'-AGC CAG GTT GGC TGT GTA-3', which was designed from the third transmembrane segment of the glutamate receptor 1 . About 7.0 $\times 10^{5}$ plaques were screened with the ${ }^{32} \mathrm{P}$ end-labelled oligonucleotide probe. The plaques were transferred onto nitrocellulose membranes, immobilized, and hybridized with hybridization solution containing the probe. The membranes were washed with $0.2 \times \mathrm{SSC} / 0.1 \%$ SDS for $10 \mathrm{~min}$ three times at room temperature and followed by at $37^{\circ} \mathrm{C}$ for $10 \mathrm{~min}$. The signal was visualized by exposure onto X-OMAT film overnight. The positive plaques were picked, and the second screening was performed as described above. The resulting two plaques, G12 and G18, were cultured and their phage DNA s were isolated. The clones were digested with Eco RI, shown to have 1.2 and $2.3 \mathrm{~kb}$ insert. Restriction mapping was carried out to make deletion mutant for the sequencing.

\section{Sequencing and sequence analysis of CDNA}

Basically the deletion mutants were prepared as previously described (Kim et al., 1998). Briefly, the 5'-/3'overhang DNA was made, and was digested with Exo III nuclease followed by $S 1$ nuclease. The unidirectional deletion DNA was ligated and transformation was carried out. The plasmids DNA from the deletion clones were prepared by Wizard miniprep kit (Promega). The template DNA for the sequencing was prepared by alkali denaturation-neutralization of the double-stranded plasmid. The sequencing was carried out by using the Sequenase v.2.0 sequencing kit (Amersham). The sequencing data from the deletion mutants were analyzed by using the MacVector program from IBI Co.

\section{Reverse transcription (RT) and PCR}

Total RNA was isolated from a 3 month-old rat cerebrum by using the acid guanidium phenol method (Chomczynski and Cacchi, 1987). Approximately $5 \mu \mathrm{g}$ of total RNA was incubated at $37^{\circ} \mathrm{C}$ for $60 \mathrm{~min}$ in $50 \mu \mathrm{l}$ volume containing 10 units of MMLV reverse transcriptase, 50 $\mathrm{mM}$ Tris- $\mathrm{HCl}, \mathrm{pH} 8.3,75 \mathrm{mM} \mathrm{KCl}, 3 \mathrm{mM} \mathrm{MgCl} 2,0.5 \mathrm{mM}$ dNTP, $0.1 \mu \mathrm{g}$ of random hexamer primers. After incubation, $10 \mu \mathrm{l}$ of the first-strand reaction mixture was amplified in a $50 \mu \mathrm{l}$ reaction mixture that included 10 pmoles of upstream primer (ASU: 5'-CAG GGG GAA TAA TGA CTG CACC-3') and of downstream primer (ASD: 5'-TGA AGG ATG TGG CTG TGT AAC C-3'), for 35 cycles $\left(10 \mathrm{sec}\right.$ at $94^{\circ} \mathrm{C}, 10 \mathrm{sec}$ at $50^{\circ} \mathrm{C}$, and $0.5 \mathrm{~min}$ at $72^{\circ} \mathrm{C}$ ) in Thermocycler Model 9600 (Perkin Elmer). The PCR products were separated on $3 \%$ agarose (Agarose : NuSieve $=2: 1$ ) gel. To verify the alternative splicing, the PCR products were cloned into T-vector and the sequencing was carried out as previously described.

\section{In situ hybridization histochemistry}

The hybridization probe for rCALM was prepared from pGEM plasmid containing $615 \mathrm{bp}$ fragment of rCALM cDNA (Pst I and Eco RI fragment, nucleotide residues 1095-1700) by transcribing with appropriate RNA polymerases using a Riboprobe System (Promega Co.) in the presence of $\alpha-\left[{ }^{35} \mathrm{~S}\right]$ UTP $(1000-1500 \mathrm{Ci} / \mathrm{mmol}$, New England Nuclear). The 645 bp-sized AP180 cDNA (nucleotide residues 2222-2866) was prepared by RT PCR of rat brain CDNA and labelled with $\left[{ }^{35}\right.$ S]UTP as above. In situ hybridization histochemistry was performed essentially as previously described (Kim et al., 1992). Briefly, frozen rat brain sections (12 $\mu \mathrm{m}$ thick) were cut, thaw-mounted onto gelatin- coated slides. The sections were fixed in $4 \%$ paraformaldehyde, treated with $0.25 \%$ acetic anhydride in $0.1 \mathrm{M}$ triethanolamine/ $0.9 \% \mathrm{NaCl}$ $(\mathrm{pH} 8.0)$ to reduce nonspecific hybridization due to electro-static force, dehydrated and defatted in ethanol and chloroform, and finally air-dried. The sections were hybridized overnight at $53^{\circ} \mathrm{C}$ with $5 \times 10^{6} \mathrm{cpm}$ of labeled RNA probe per slide. Then the sections were washed in 2x SSC, treated with RNase A $(20 \mathrm{mg} / \mathrm{ml}$, BoehringerMannheim) for $30 \mathrm{~min}$ at $37^{\circ} \mathrm{C}$ and washed sequentially for $60 \mathrm{~min}$ in $2 \mathrm{x} \mathrm{SSC}$ at $50^{\circ} \mathrm{C}, 60 \mathrm{~min}$ in $0.2 \mathrm{xSC}$ at $55^{\circ} \mathrm{C}$, and $60 \mathrm{~min}$ in $0.2 \times \mathrm{SSC}$ at $60^{\circ} \mathrm{C}$. After drying, the slides were processed for film ( $\beta$-max Hyperfilm, Amershampharmacia) autoradiography by exposing 4 days.

\section{Results and Discussion}

\section{Primary structure of clathrin assembly protein from rat (rCALM)}

The clathrin assembly protein gene was found from the rat brain cDNA library by low stringency screening using the oligonucleotide designed from the third transmembrane segment of the glutamate receptor 1 . Among 48 cDNA clones, two clones, G12 and G18, were shown to have high homology with N-terminal region of AP180 and CALM when they were searched with blast program of $\mathrm{NCBI}$ at $\mathrm{NIH}$. The size of $\mathrm{G} 12$ and $\mathrm{G} 18$ were 1.2 and $2.3 \mathrm{~kb}$ respectively. G18 encompasses a full coding region sequences from putative translation initiation 
GGGGTGGCGGACGAGCTGCAGAG ATG TCT GGC CAG AGC CTG ACG GAC CGA ATC ACC GCG GCC CAG 65 Met Ser Gly GIn Ser Leu Thr Asp arg Ile Thr Ala Ala GIn

CAC AGT GTC ACT GGC TCC GCG GTA TCT AAG ACA GTA TCC AAG GCC ACG ACC CAC GAG ATC 125 His Ser Val Thr Gly Ser Ala Val Ser Lys Thr Val Cys Lys Ala Thr Thr His Glu lle 34 ATG GGC CCC AAG AAA AAG CAC CTG GAC TAC TTA ATT CAG TGT ACA AAT GAG ATG AAT GTG 185 Met Gly Pro Lys Lys Lys His Leu Asp Tyr Leu lle Gin Cys Thr Asn Glu Met Asn Val 54 AAT ATC CCA CAG TTG GCA GAC AGT TTG ITT GAA AGA ACT ACT AAT AGT AGT TGG GTG GTG 245 Asn Ile Pro Gin Leu Ala Asp Ser Leu phe Glu Arg Thr Thr Asn Ser Ser Trp Val Val 74 GTC TTC AAA TCA CTC ATT ACA ACT CAT CAT TIG ATG GTG TAT GGA AAC GAG CGT TTC ATT 305 Val Phe Lys Ser Leu Ile Thr Thr His His Leu Met Val Tyr Gly Asn Glu Arg Phe Ile 94

CAG TAT TTG GCT TCA AGA AAC ACA TTG TIT AAC TTA AGC AAC TTT TTG GAT AAA AGT GGA 365 GIn Tyr Leu Ala Ser Arg Asn Thr Leu Phe Asn Leu Ser Asn Phe Leu Asp Lys Ser Gly 114

ITG CAA GGA TAT GAT ATG ICT ACA TIT ATT AGA CGA TAT AGT AGG TAC CTA AAT GAA AAG 425 Leu Gin Gly Tyr Asp Met Ser Thr Phe Ile Argarg Tyr Ser Arg Tyr Leu Asn Glu Lys 134 GCA GTT TCA TAC AGA CAA GTT GCA TTC GAT IIC ACA AAA GTG AAG AGA GGA GCT GAT GGA 485 Ala Val Ser Tyr Arg GIn Val Ala Phe Asp Phe Thr Lys Val Lys Arg Gly Ala Asp Gly 154 GT ATG AGA ACA ATG AAC ACA GAA AAA CTG TAA AAA ACT GTA CCA ATT ATC CAA AAT CAA 545 Val Met Arg Thr Met Asn Ihr Glu Lys Leu Leu Lys Thr val Pro Ile Ile GIn Asn GIn 174 ATG Met Asp Ala Leu Leu Asp phe Asn Val Asn Ser Asn Glu Leu Thr Asn Gly Val Ile Asn 194

GCT GCC TTC ATG CTC CTG ITC AAA GAT GCC ATT AGA CTA ITT GCA GCA TAC AAT GAA GGA 665 Ala Ala Phe Met Leu Leu Phe Lys Asp Ala lle Arg Leu Phe Ala Ala Tyr Asn Glu Gly 214

ATT ATT AAT TIA TTG GAA AAA TAT TIT GAT ATG AAA AAG AAC CAG TGC AAA GAA GGT CTT 725 Ile Ile Asn Leu Leu Glu Lys Tyr Phe Asp Met Lys Lys Asn GIn Cys Lys Glu Gly Leu 234

GAC ATC TAT AAG AAG TIT ITG ACT AGG ATG ACA AGA ATC TCA GAG TIT CTG AAA GTT GCA 785 Asp Ile Tyr Lys Lys Phe Leu Thr Arg Met Thr Arg Ile Ser Glu Phe Leu Lys Val Ala 254

GAG CAA GTT GGA ATT GAC AGA GGA GAT ATT CCA GAT CTT TCA CAG GCC CCC AGC AGT CTT 845 Glu GIn Val Gly Ile Asp Arg Gly Asp Ile Pro Asp Leu Ser Gln Ala Pro Ser Ser Leu 274 CTT GAT GCT TIA GAA CAA CAT TTA GCT TCC TTG GAA GGG AAG AAA ATC AAA GAT TCC ACA 905 Leu Asp Ala Leu Glu GIn His Leu Ala Ser Leu Glu Gly Lys Lys Ile Lys Asp Ser Thr 294

GCT GCA AGC AGG GCT ACA ACA CTT TCC AAT GCA GTC TCT TCT TTG GCA AGC ACT GGC CTA 965 Ala Ala Ser Arg Ala Thr Thr Leu Ser Asn Ala Val Ser Ser Leu Ala Ser Thr Gly Leu 314 TCT CTG ACC AAA GTG GAT GAA AGG GAA AAG CAG GCA GCA TTA GAG GAA GAA CAG GCT CGA 1025 Ser Leu Thr Lys Val Asp Glu Arg Glu Lys GIn Ala Ala Leu Glu Glu Glu Gin Ala Arg 334
ITA AAA GCA CTA AAG GAA CAG CGT CTA AAA GAA CTT GCA AAG AAA CCG CAT ACC TCT TTA 1085 Leu Lys Ala Leu Lys Glu GIn Arg Leu Lys Glu Leu Ala Lys Lys Pro His.Thr Ser Leu 354 ACA ACT GCA GCC TCT CCT GTG TCC ACC TCA GCA GGG GGA ATA ATG ACT GCA CCA GCC ATC 1145 Thr Thr Ala Ala Ser Pro val Ser Thr Ser Ala Gly Gly lle Met Thr Ala pro Ala lle 374 GAC ATA TIT TCT ACC CCT AGT TCT TCT AAC AGC ACA TCC AAG CTG CCA AAT GAC CTG CTT 1205 Asp Ile Phe Ser Thr Pro Ser Ser Ser Asn Ser Ihr Ser Lys Leu Pro Asn Asp Leu Leu 394 GAT TTG CAG CAG CCA ACC TIT CAT CCA TCT GTC CAT GCT ATG TCA GCT GCT CCT CAG GTA 1265 Asp Leu Gln GIn Pro Thr Phe His Pro Ser Val His Ala Met Ser Ala Ala Pro Gin Val 414

GCA AGT ACA TGG GGA GAT GCT GTT GAT GAT GCC ATT CCA AGC TTA AAT CCT TTC CTC ACA 1325 Ala Ser Thr Trp gly Asp Ala Val Asp Asp Ala Ile Pro Ser Leu Asn Pro Phe Leu Thr 434

AMA AGT AGT GGT GAT GTT CAC CTT CCT ATT TCT TCA GAT GTA TCC ACT TTT ACT ACT AGG 1385 Lys Ser Ser Gly Asp Val His Leu Pro lle Ser Ser Asp Val Ser Thr Phe Thr Thr Arg 454

ACA CCT ACT CAT GAA ATG TTT GTT GGA TTC AGT CCT TCT CCGG GT ACA CAG CCA CAT CCT 1445 Thr Pro Thr His Glu Met Phe Val Gly Phe Ser Pro Ser Pro Val Thr GIn Pro His Pro 474 ICA GCT GGC CTT AAT GTT GAC IIT GAA TCT GTG IIT GGA AAT AAG TCT ACG AAT GTT GCT 1505 Ser Ala Gly Leu Asn Val Asp Phe Glu Ser Val Phe Gly Asn Lys Ser Thr Asn Val Ala 494 GTA GAT TCT GGT GGT GGA CTT CTC AAA CCA ACA GTG GCC TCT CAG AAC CAG AGT CTT CCT 1565 Val Asp Ser Gly Gly Gly Leu Leu Lys Pro Thr Val Ala Ser GIn Asn GIn Ser Leu Pro 514

GTT GCC AAA CTT CCG CCT AAC AAA TTA GTG TCT GAT GAC TTG GAT TCA TCT TTA GCC AAC 1625 Val Ala Lys Leu Pro Pro Asn Lys Leu Val Ser Asp Asp Leu Asp Ser Ser Leu Ala Asn 534

CTT GTG GGC AAT CTT GGC ATT GGA AAT GGA ACC ACT AAG AAT GAT GTA AGT TGC AGT CAA 1685 Leu Val Gly Asn Leu Gly Ile Gly Asn Gly Thr Thr Lys Asn Asp Val Ser Cys Ser Gln 554 CCA GGT GAA AAG AAG TTA ACT GGA GGA TCT AAC TGG CAA CCA AAG GTC GCA CCA ACA ACT 1745 Pro Gly Glu Lys Lys Leu Thr Gly Gly Ser Asn Trp GIn Pro Lys Val Ala Pro Thr Thr 574 GCC TGG AGT GCT GCA ACA ATG GCA CCC CCT GTA ATG GCC TAT CCT GCT ACT ACA CCA ACG 1805 Ala Trp Ser Ala Ala Thr Met Ala Pro Pro Val Met Ala Tyr Pro Ala Thr Thr Pro Thr 594

GGC ATG ATA GGA TAT GGA ATT CCT CCT CAG ATG GGA AGT GTA CCT GTA ATG ACA CAG CCA 1865 Gly Met Ile Gly Tyr Gly Ile Pro Pro GIn Met Gly Ser Val Pro Val Met Thr GIn Pro 614

ACC TTA ATA TAC AGC CAG CCT GTC ATG AGA CCG CCA AAC CCC ITT GGC CCT GTA CCA GGA 1925 Thr Leu Ile Tyr Ser GIn Pro Val Met Arg Pro Pro Asn Pro Phe Gly Pro Val Pro Gly 634 GCA CAG ATA CAG TIT ATG TAA CTAGATGGAagagaAtgGaATtACTCCAAGAATAGAaGTGCACAGGTGGCG 1997 Ala Gin I le GIn Phe Met $* * *$

ACTCCTIACTTCCAGCAAAATCCAAACTGCTGTCTCTAAGACTCTTCCTCC 2048

Figure 1. Complete nucleotide and deduced amino acid sequence of the rat CALM. Numbers refer to nucleotide and amino acid positions. Beneath the nucleotide sequence is the deduced amino acid sequence coded for by the open reading frame between nucleotide 24 and 2048 . The alternatively spliced region is shown as bold. The arrows indicate the primers (ASU and ASD) to verify the alternatively splicing sequences. The possible phophorylation sites are; casein kinase II (S-X-X-D/E, shown as open circles), cAMP-dependent proein kinase A (R-R-X-S, closed circle), protein kinase $C\left(S / T-X-R / K\right.$, open square), Ca ${ }^{2+} /$ calmodulin-dependent protein kinase (R-X-X-S/T, closed square). The above sequence has been deposited in the GenBank database under the accession number AF041373 (short form) and AF041374 (long form).

methionine (ATG) at position 30 through termination codon (TAA) located at position 1946 (Figure 1). The 3'untranslated region (3'-UTR) terminates with a polyA tail preceded by an AAUAAA polyadenylation signal. Analysis of the deduced amino acid sequences of the rCALM did not reveal a signal sequences, or any regions of extensive hydrophobicity. G18 contains a full open reading frame encoding the clathrin assembly protein (short form of rCALM) with 597 amino acids. Because the nucleotide sequence of rCALM shows $129 \mathrm{bp}$-short comparing to that of CALM, PCR was carried out to determine whether alternative splicing exists in rCALM. The rCALM with 640 amino acids was shown to have an alterntive splicing variant with 129 nucleotide insert at the middle of the transcript (long form of rCALM). Estimated molecular masses of short form and long form of rCALM are 63,792 and 69,280 , respectively.

The primary structure of the rCALM, shows a remarkable homology to the murine and rat clathrin assembly protein AP180 (Zhou et al., 1992; Morris et al., 1993;
Zhou et al., 1993) (Figure 2). The homology ranges from $97 \%$ to $28 \%$ in different portions of the protein. The most striking homology of more than $95 \%$ is found between the first 289 amino acids of rCALM and that of AP180. The rCALM protein was 256 amino acid short compared to AP180, the midlle part of which is missing in rCALM. The amino acid sequences of the C-terminal half of the rCALM is different from that of AP180. The overall pl values of the rCALM and AP180 were 8.7 and 4.6, respectively. The missing 250 amino acids which correspond to the acidic middle region of AP180 caused the pl value of rCALM alkaline. AP180 is interrupted by acidic and proline rich middle domains.

The distribution of charged amino acids, proline and alanine suggest a two-domain structure of rCALM in contrast to the three-domain structure of AP180. The Nterminal region of 340 amino acids is highly charged and predominantly basic. Twenty five percent of the amino acids in the region is charged, $14 \%$ of which is basic amino acids. Especially the first 289 residues are 
rCALM MSGQSLTDRI TAAQHSVTGS AVSKTVCKAT THEIMGPKKK HLDYLIQCTN 50 hCALM MSGQSLTDRI TAAQHSVTGS AVSKTVCKAT THEIMGPKKK HLDYLIQCTN 50 AP180 MSGQtLTDRI aAAQySVTGS AVaraVCKAT THEVMGPKKK HLDYLIQaTN 50

rCALM EMNVNIPQLA DSLFERTTNS SWVVVFKSLI TTHHLMVYGN ERFIQYLASR 100 hCALM EMNVNIPQLA DSLFERTTNS SWVVVFKSLI TTHHLMVYGN ERFIQYLASR 100 AP180 EtNVNIPQmA DtLFERaTNS SWVVVFKaLV TTHHLMVhGN ERFIQYLASR 100

rCALM NTLFNLSNFL DKSGLQGYDM STFIRRYSRY LNEKAVSYRQ VAFDFTKVKR 150 hCALM NTLFNLSNFL DKSGLQGYDM STFIRRYSRY LNEKAVSYRQ VAFDFTKVKR 150 AP180 NTLFNLSNFL DKSGShGYDM STFIRRYSRY LNEKAfSYRQ mAFDFarVKk 150

rCALM GADGVMRTMN TEKLLKTVPI IQNOMDALLD FNVNSNELTN GVINAAFMLL 210 hCALM GADGVMRTMN TEKLLKTVPI IONOMDALLD FNVNSNELTN GVINAAFMLL 210 AP180 GADGVMRTMV pEKLLKsmPI IQgQiDALLe FdVhPNELTN GVINAAFMLL 210

rCALM FKDAIRLFAA YNEGI INLLE KYFDMKKNOC KEGLDIYKKF LTRMTRISEF 250 hCALM FKDAIRLFAA YHEGI INLLE KYFDMKKNOC KEGLDIYKKF LTRMTRISEF 250 AP180 FKDI IKLFAC YNdGvINLLE KfFeMKKgQC KdnLeIYKrF LTRMTRvSEF 250

rCALM LKVAEQVGID RGDIPDLSQA PSSLLDALEQ HLASLEGKKI KDSTAASRAT 300 hCALM LKVAEQVGID RGDIPDLSQA PSSLLDALEQ HLASLEGKKI KDSTAASRAT 300 AP180 LKVAdeVGID kGDIPDLtQA PSSLmetLEQ HLatLEGKK. ......... 289

rCALM TLSNAVSSLA STGLSLTKVD EREKQAALEE EQARLKALKE ORLKELAKK. 349 hCALM TLSNAVSSLA STGLSLTKVD EREKQAALEE EQARLKALKE ORLKELAKK. 349

AP180 pgnnegsgap sp/sksspat tvtspnstpa ktidtsppvd ifatasaaap 339

AP180 vssakpssdI |d|qpdfsga ragaaapvpp ptggatawgd I|geds|aal 389 AP180 ssvpseapis dpfapepspp ttttepasas asattavtaa ttevdlfgda 439 AP180 faaspgeapa asegatapat papvaaalda csgndpfaps egsaeaapel 489
rCALM PHTSLTTAAS .P. VSTSAGG 367

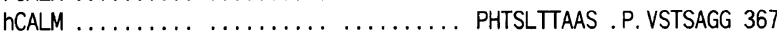
AP180 tttattsaaa attaaappal difgdlfdsa Pe..va. AAS kPdV... Aps 583

rCALM IMTAPAIDIF STP... SSSN STSKLPNDLL DLQQPTFHPS VHAMSAAPQV 414 hCALM IMTAPAIDIF STP... SSSN STSKLPNDLL DLQQPTFHPS VHPMSTASQV 414 AP180 I. dl fgtDaF SsPprgaSpv peSsLtaDLL ......... S VdAf. AAPsp 622

rCALM ASTWG---. . .----DAVD. DAIPSLNPFL TKSSGDVHL. PISSDVSTFT 452 hCALM ASTWGdpf. . . satVDAVD. DAIPSLNPFL TKSSGDVHL. SISSDVSTFT 459 AP180 ASTas paka essgviDifg DA. .....Fg . .. SSasetq PapqaVSsss 662

rCALM TRTPTHEMFV G. F. SPS. .P VTQPHPSAGL .. NVDFESVF GNK. STNVAV 495 hCALM TRTPTHEMFV G. F. TPS. . P VaQPHPSAGL .. NVDFESVF GNK. STNV iV 502 AP180 asadl lagFg GsFmaPSttP VT.P. aqnnL qpN. . FEaaF GttpST. sss 707

rCALM VTQPHPSAGL .. NVDFESVF GNK. STNVAV DSG-----GG LLKPTVASQN 510 hCALM VaQPHPSAGL .. NVDFESVF GNK. STNViV DSGgfdeIGG LLKPTVASQN 522 AP180 VT. P. aqnnL qpN. . FEaaF GttpST. sss sS. . fdpsGd LLmPTmApsg 726

rCALM QSLPVAKLPP ....... NK. L VSDDLDSSLA NLVGNLGIGN GTT.KN. DVS 551 hCALM OnLPVAKLPP ...... sK.L VSDDLDSSLA NLVGNLGIGN GTT. KN. DVn 563 AP180 QpaPVsmvPP spamsasKgL gSD. LDSSLA sLVGNLGIs. GTTsKkgDIq 774

rCALM CSQPGEKKLT GGSNWQPKVA PTTAWSAATM APPVMAYPAT TPT...... 594 hCALM WSQPGEKKLT GGSNCEPKVA PTTAWNAATM APPVMAYPAT TPT...... 606 AP180 w. naGEKKLT GGaNWQPKVt PaT. WSAgv. .PPqgtvP. . .PTssvppga 817

rCALM ........GM IGYGIPPQ. . ... MGSVPV MTQPTLIYSQ PVMRPPNPFG 630 hCALM ........GM IGYGIPPQ. . ... MGSVPV MTQPTLIYSQ PVMRPPNPFG 642 AP180 gapsvgqpG. aGYGmPPagt gmtmMpQqPV Mfa...... P PMRPP. . FG 858

rCALM P. . VPG.... .................. AQI. OFM* hCALM P. VSG.... .................. AQI. QFM* AP180 aaaVPGtQls psptpatasp kkppakdpla d|n|kdF|*

AP180 dlfamkppet sapvvtptas tappvpatap spaptavaat aatttaaaaa 539

Figure 2. Amino acid sequence comparison between the rat CALM and the human CALM and rat AP180. There is high degree of homology(more than $90 \%$ ) between the genes from amino acids 1 to 289. The interrupted dots show the very different region, and the low degree of identity (around $30 \%$ ) was shown at the C-terminal region. Differences between the sequences are indicated as small letter. The dashed line shows the missing of the amino acids and asterisk shows the translation-termination of amino acid.

almost identical to the corresponding sequences of AP180 from rat. Functional studies of the AP180 have shown that a clathrin-binding domain resides in the 33 $\mathrm{kD} \mathrm{N}$-terminal portion of the protein. This $33 \mathrm{kD}$ region is thought to have a globular structure and consists mainly of helices (Ye and Lafer, 1995). Although this Nterminal domain is able to bind clathrin triskelia, it is unable to assemble them into clathrin cages and bind to preassembled cages. The assembling function resides in the $58 \mathrm{kD}$ C-terminal region of the AP180. The high conservation of $\mathrm{N}$-half amino acid of the rCALM suggests that the protein has similar clathrin binding properties. Another important function of the $33 \mathrm{kD} \mathrm{N}$-terminal region is its high affinity binding of both inositol hexakisphosphate and diphosphoinositol pentakisphosphate (Norris et al., 1995; Ye et al., 1995). Binding of these ligands inhibits the ability of AP180 to assemble clathrin into cages (Ye et al., 1995). The binding site for the inositide was suggested to be KKK at $\mathrm{N}$-terminal side, that is well conserved in rCALM and AP180. Thus, inositol polyphosphates and phosphatidyl inositides may regulate clathrin-coated vesicle assembly and/or disassembly by means of the monomeric clathrin assembly proteins,
rCALM and AP180. Prasad (1995) reported that myelin basic protein would be a novel clathrin assesmbly protein. The protein has $23 \%$ of charged amino acids, and 30 out of 38 charged amino acids are basic. The highly charged and basic character of $\mathrm{N}$-half of the rCALM is similar with that of myelin basic protein.

Analysis of deduced amino acid composition revealed an unusually large number of proline residues ( 45 prolines 1640 residues). Most (36 out of 45 ) of the prolines are localized at the C-terminal half of the rCALM, where the proline levels approach $12.5 \%$, while the proline composition is just $1.6 \%$ at the $\mathrm{N}$-terminal half. In the case of AP180, which has three domain structures, the middle and C-terminal domain is rich in proline (15\%). There are multiple PXXP amino acid sequences, which is a known ligand for $\mathrm{SH} 3$ domain. Amphiphysin and other proteins containing $\mathrm{SH} 3$ domains would participate in the regulation of the clathrin cage foramtion. The polyproline could be the ligand for the WW domain. Although the typical sequence of XPPPXY was not found in the rCALM, there are XPPX sequences that could bind WW domain. Considering the role of WW domain containing protein, such as dystrophin, in the 
Std $\mathrm{cr}$ cl ht in lv lu kd sp pn ts th sg sm

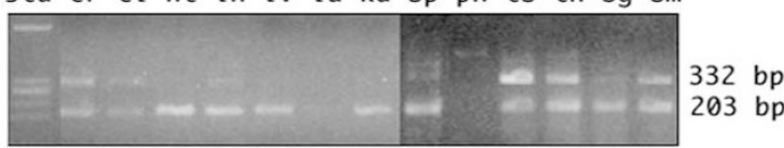

Figure 3. Differential expression of the clathrin assembly protein transcript in various tissues of rat. The oligonucleotides used for PCR are indicated by arrows in Figure 1. The alternative splicing sites were amplified from the mRNA of adult rat tissues by using RT PCR as described in 'Materials and Methods', and analyzed on $3 \%$ agarose (Agarose: NuSieve $=2: 1$ ) gel. The left lane indicates $\Phi X 174 \mathrm{Hae}$ III size marker. The sizes of the upper and lower band are $332 \mathrm{bp}$ and $203 \mathrm{bp}$ respectively. The tissues from the left are: cr, cerebrum, cl; cerebellum, ht; heart, in; intestine, lv; liver, lu; lung, kd; kidney, sp; spleen, pn; pancrease, ts; testis, th; thymus, sg; salivary gland, sm; skeletal muscle.

interaction between cytoskeleton and membrane structure (Sudol, 1996), the rCALM and AP180 are important in the transport of vesicle through the bridge-formation between cytoskeleton and CCV. The possible function of the marked different region of the C-terminal half could be an interaction site(s) with proteins in the plasma membrane, possibly contributing to the regulation of the endocytotic activity and receptor turnover of the cell.

There are eight possible phosphorylation sites; three casein protein kinase II and protein kinase $\mathrm{C}$, one cAMP-dependent protein kinase $\mathrm{A}$, and one $\mathrm{Ca}^{2+} /$ calmodulin kinase. Except one protein kinase $C$ site, most of the kinase substrate sites are located on the $\mathrm{N}$ terminal half of the protein. where prolines and alanines are rare. The AP180 protein was known as a neuronspecific phosphoprotein (Keen and Black, 1986). High degree of homology of rCALM with AP180 suggested that the rCALM would be phosphorylated in vivo. The major role of AP180 would be the formation of uniformsized vesicle in the nervous system (McMahon, 1999). Considering the ubiquitous expression of the rCALM and inhibitory activity of phosphorylated AP-1 and AP-2 (Wilde and Brodsky, 1996) on the formation of clathrin cage, the phosphorylation of the rCALM would be involved in the regulation of the uniform-sized clathrin cage formation in non-nervous systems.

\section{Alternatively splicing}

To determine the differential expression of the alternatively splicing variants, RT-PCR was carried out. The difference between the long and short form of rCALM was 129 nucleotide insertion or deletion by alternatively splicing. The short form was shown as 203 bp-sized PCR product, that was observed in most of the tissues examined (Figure 3). The $332 \mathrm{bp} \mathrm{PCR} \mathrm{product,} \mathrm{the} \mathrm{long} \mathrm{form} \mathrm{of}$ the rCALM, was not observed in heart, lung, and pancreas. The rCALM might have a complementary role in the formation of uniform-sized CCV in neural and non-neural tissues. But there was no functional domains so far known in the alternatively spliced segment. The
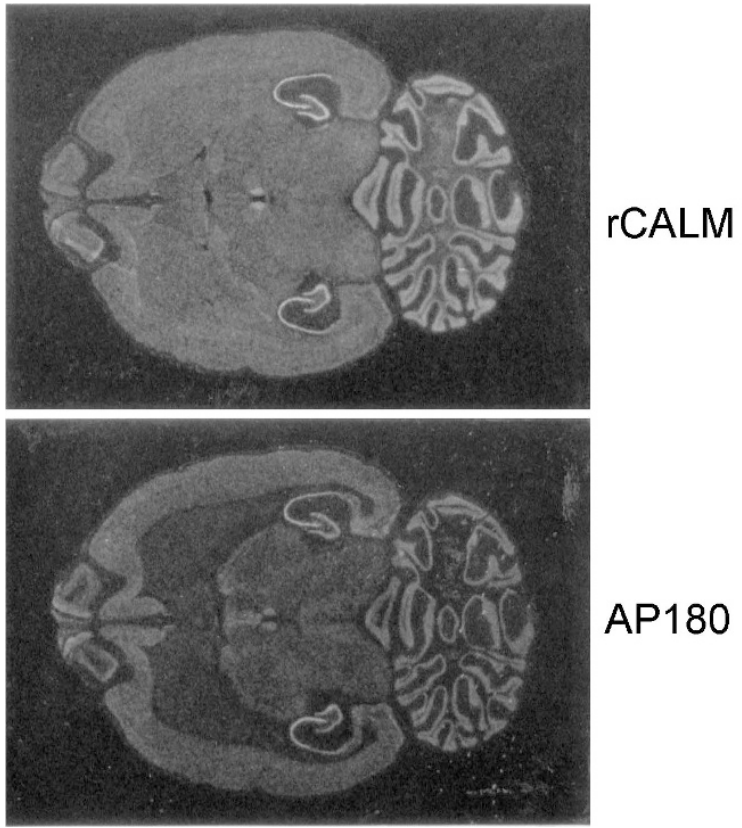

Figure 4. Localization of rCALM and AP180 in adult rat brain by in situ hybridization. Both figures show the negative film image of the expression of rCALM (shown upper) and AP180 (lower) in the transverse section of adult rat brain. In situ hybridization was carried out as described in 'Materials and Methods'.

exact functional role of the differential expression remains to be studied.

\section{Expression in adult rat brain}

The expression pattern of the rCALM gene was determined in adult rat brain by in situ hybridization histochemistry. The rCALM riboprobe was 615 bp-sized Pst $\mathrm{I}$ and Eco RI fragment (nucleotide residues 1,095-1700) from G18 cDNA, which encodes a unique sequence compared to that of other types of assembly proteins, in order to avoid a cross reaction with other assembly protein mRNAs. The riboprobe of AP180 was also designed from the unique 3 '-end lower one third sequences. To establish the specificity of the labelled probe, control experiments were conducted as follows. First, a control hybridization using a sense probe resulted in autoradiograms virtually devoid of signal. Second, hybridization signal from the tissue was abolished by addition of RNase in the hybridization solution. Figure 4 shows the transverse section of the adult rat brain. The expression of the rCALM mRNA was more intense than that of AP180 and the distribution pattern was different each other. The rCALM mRNA was widely expressed throughout the brain. High levels of rCALM were found on the hippocampus, dentate gyrus, medial habenula nucleus, and cerebellar granule cells. Relatively high levels of expression were observed in olfactory bulb, and cerebral cortex. Moderate densities were in the 
caudate-putamen, thalamus, and superior colliculus. The AP180 gene was expressed moderately in cerebral cortex, olfactory bulb, hippocampus, dentate gyrus, and cerebellar cortex, while it was faintly expressed in caudate-putamen. From this different distribution pattern, it may be suggested that these two monomeric clathrin assembly proteins function differently; AP180 would work as a neuronal synaptic vesicle assembly protein, and rCALM might assemble endocytic vesicles in neural and supporting cells in brain.

\section{Acknowledgement}

I thank Hye-Sun Jung at Dept. of Anatomy, Korea University for her excellent technical assistance of in situ hybridization. This study was supported by the Grant of the Korea Research Fund, Basic Medical Science (997021-F0019).

\section{References}

Chomczynski, P. and Sacchi, N. (1987) Singl-step method of RNA isolation by acid guanidium thiocyanate-phenol-chloroform extraction. Anal. Biochem. 162: 156-159

Dell'Angelica, E. C., Mullins, C. and Bonifacino, J. S. (1999) AP-4, a novel protein complex related to clathrin adaptors. $J$. Biol. Chem. 274: 7278-7285

Keen, J. H. and Black, M. B. (1986) The phosphorylation of coated membrane proteins in intact neurons. J. Cell Biol. 102: $1325-1333$

Keen, J. H. (1990) Clathrin associated assembly and disassembly proteins. Ann. Rev. Biochem.. 59: 415-438

Kim, H.-L., Chang, Y.-J., Lee, S.-M. and Hong, Y.-S. (1998) Genomic structure of the regulatory region of the voltagegated calcium channel 1D. Exp. Mol. Med. 30: 246-251

Kim, H.-L., Kim, H., Lee, P., King, R. G. and Chin, H. (1992) Rat brain expressed an alternatively spliced form of the dihydropyrdine-sensitive L-type calcium channel a2 subunit. Proc. Nat'l Acad. Sci. USA. 89: 3251-3255

Kondury, P. and Roland, E. L. (1988) Molecular characterization of the AP180 coated vesicle assembly protein. Biochem. 27: $6098-6104$

Marsh, M. and McMahon, H. T. (1999) The structural era of endocytosis. Science 285: 215-219
McMahon, H. T. (1999) An assembly protein for clathrin cages. Curr. Biol.. 9: R332-R335

Morris, S. A., Schroder, S., Plessmann, U., Weber, K. and Ungewickell, E. (1993) Clathrin assembly protein AP 180: primary structure, domain organization and identification of a clathrin binding site. The EMBO J. 12: 667-675

Norris, F. A., Ungewickell, E. and Majerus, P. W. (1995) Inositol hexakisphosphate binds to clathrin assembly protein 3(AP-3/AP180) and inhibits clathrin cage assembly in vitro. J. Biol. Chem. 270: 214-217

Pearse, B. M. and Robins, M. S. (1990) Clathrin,adaptors, and sorting. Ann. Rev. Cell. Biol. 6: 151-171

Prasad, K., Barouch, W., Martin, B. M., Greene, L. E. and Eisenberg, E. (1995) Purification of new clathrin assembly protein from bovine brain coated vesicles and its identification as myelin basic protein. J. Biol. Chem. 270: 30551-30556

Robinson, M. S. (1994) The role of clathrin, adaptors, and dynamin in endocytosis. Curr. Opin. Cell. Biol. 6: 538-544

Simpson, F., Peden, A. A., Christopoulou, L. and Robinson, M. S. (1997) Characterization of the adaptor-related protein complex, AP-3. J. Cell Biol. 137: 835-845

Stephen, A. M., Annette, M. and Ungewickell, E. (1990) Anaysis of 100-180 kD phosphoprotein in clathrin-coated vesicles from bovine brain. J. Biol. Chem. 265: 3354-3357

Sudol, M. (1996) The WW domain binds polyproline and is involved in human diseases. Exp. Mol. Med. 28: 65-69

Wilde, A. and Brodsky, F. M. (1996) In vivo phophorylation of adaptors regulates their interaction with clathrin. J. Cell Biol. 135: $635-645$

Ye, W., Ali, N., Bembenek, M. E., Shears, S. B. and Lafer, E. M. (1995) Inhibition of clathrin assembly by high affinity binding of specific inositol polyphosphate to the synapsespecific clathrin assembly protein AP-3. J. Biol. Chem. 270: 1564-1568

Ye, W. and Lafer, E. M. (1995) Clathrin binding and assembly activities of expressed domains of the synapse-specific clathrin assembly protein AP-3. J. Biol. Chem. 270: 10933-10939

Zhou, S., Sousa, R. and Lafer, E. M. (1992) Characterization of a novel synapse-specific protein II. cDNA cloning and sequence analysis of F1-20 protein. J. Neurochem. 12: 21142155

Zhou, S., Tannery, N. H. and Lafer, E. M. (1993) The synapsespecific phosphoprotein $\mathrm{F} 1-20$ is identical to the clathrin assembly protein AP-3. J. Biol. Chem. 268: 12655-12662 